\title{
Einstein boundary conditions for the Einstein equations in the conformal-traceless decomposition
}

\author{
Simonetta Frittell ${ }^{\circledast}$ \\ Department of Physics, Duquesne University, Pittsburgh, PA 15282 and \\ Department of Physics and Astronomy, University of Pittsburgh, Pittsburgh, PA 15260 \\ Roberto Gómedt \\ Pittsburgh Supercomputing Center, Carnegie Mellon University, Pittsburgh, PA 15213, and \\ Department of Physics and Astronomy, University of Pittsburgh, Pittsburgh, PA 15260
}

(Dated: October 30, 2018)

\begin{abstract}
In relation to the BSSN formulation of the Einstein equations, we write down the boundary conditions that result from the vanishing of the projection of the Einstein tensor normally to a timelike hypersurface. Furthermore, by setting up a well-posed system of propagation equations for the constraints, we show explicitly that there are three constraints that are incoming at the boundary surface and that the boundary equations are linearly related to them. This indicates that such boundary conditions play a role in enforcing the propagation of the constraints in the region interior to the boundary. Additionally, we examine the related problem for a strongly hyperbolic first-order reduction of the BSSN equations and determine the characteristic fields that are prescribed by the three boundary conditions, as well as those that are left arbitrary.
\end{abstract}

PACS numbers: 04.25.Dm, 04.20.Ex

\section{INTRODUCTION}

In a series of papers [1, 2, $[3]$ we have carried out a study of the relevance of the projection of the Einstein tensor $G_{a b}$ perpendicularly to a timelike surface as candidate boundary conditions for the initial-boundary value problem of the Einstein equations. Using the EinsteinChristoffel formulation [4] we demonstrated that the vanishing of three of the four components of the projection $G_{a b} e^{b}$ (where $e^{b}$ is the vector normal to the timelike boundary) are the three necessary and sufficient boundary conditions required for the initial value problem, and that they are also equivalent to the three necessary and sufficient boundary conditions required for the well-posed propagation of the vanishing of the constraints. Because of the generality of the methods used to demonstrate this result, we believe that such will be the case for any formulation of the $3+1$ Einstein equations that is strongly hyperbolic.

The full significance of $G_{a b} e^{b}=0$ to the more standard $3+1$ formulation referred to as ADM [5] is not as well established, essentially due to the lack of hyperbolicity of the ADM equations themselves. Yet one can see that failure to impose at least one of the four equations leads to constraint violations in the solution at later times [3].

At this time, we are intersted in investigating the relevance of the Einstein boundary conditions $G_{a b} e^{b}=0$ to the "conformal-traceless" decomposition of the $3+1$ equations due to Baumgarte and Shapiro [6], which is developed on the basis of earlier work by Shibata and

\footnotetext{
*Electronic address: simo@mayu.physics.duq.edu
}

†Electronic address: gomez@psc.edu
Nakamura 7], and is known as BSSN. We have two reasons for interest in this formulation. In the first place, it is a hybrid between the ADM equations and the more recent hyperbolic formulations, because it represents a partial reduction to first order of the original ADM equations with mixing of the constraints in a manner similar to most hyperbolic formulations (see, e.g., [ 8$]$ ). As such, it represents an opportunity to investigate the transition between ADM and its hyperbolic (well-posed) reductions.

Our other reason for interest stems from the fact that numerical simulations performed using codes based on the BSSN formulation appear to be able to run for significantly long times, which makes it, currently, a formulation of widespread choice in numerical relativity (see, e.g., [9]), up to slight modifications (see, e.g., 10, 11]).

In Section [11 we briefly describe the problem of the Einstein boundary conditions for the ADM equations with the purpose of introducing the Einstein boundary conditions in terms of the fundamental variables of the $3+1$ split. The Einstein boundary conditions in terms of the BSSN variables are introduced in Section IIII whereas their relationship to the propagation of the constraints is developed in Section [IV] In Section $[\nabla$ we study the analogous problem for the case of a well-posed first-order reduction of the BSSN equations. Our first-order reduction is strongly hyperbolic, having six characteristic fields that are incoming at the boundary, and six that are outgoing at the boundary. Unlike the Einstein-Christoffel formulation and other first-order reductions of the BSSN equations, it has two subluminal characteristic speeds in addition to light speed and rest. We show explicitly how the Einstein boundary conditions prescribe only three of the incoming characteristic fields, which is entirely consistent with the results of Section IV] and coincides with our previous results on the Einstein-Christoffel formula- 
tion. As in our previous work, we restrict to the case of vanishing shift vector throughout. We close in Section VI with some remarks.

\section{THE EINSTEIN BOUNDARY CONDITIONS IN TERMS OF THE INTRINSIC AND EXTRINSIC CURVATURE FOR THE CASE OF VANISHING SHIFT VECTOR}

This section develops a brief review of the boundary conditions of the Einstein equations in the ADM formulation. In our minds, the ADM case acts as the basis for most other formulations that are derived from it by linear combinations with the constraints and by the addition of any number of first-order variables.

Throughout the article we assume the following form for the metric of spacetime in coordinates $x^{a}=\left(x^{i}, t\right)$ in terms of the three-metric $\gamma_{i j}$ of the slices at fixed value of $t$ :

$$
d s^{2}=-\alpha^{2} d t^{2}+\gamma_{i j} d x^{i} d x^{j}
$$

where $\alpha$ is the lapse function. The Einstein equations $G_{a b}=0$ for the four-dimensional metric are equivalently expressed in the ADM form [5]:

$$
\begin{aligned}
\dot{\gamma}_{i j} & =-2 \alpha K_{i j}, \\
\dot{K}_{i j} & =\alpha\left(R_{i j}-2 K_{i l} K^{l}{ }_{j}+K K_{i j}\right)-D_{i} D_{j} \alpha,
\end{aligned}
$$

with the constraints

$$
\begin{aligned}
\mathcal{C} & \equiv \frac{1}{2}\left(R-K_{i j} K^{i j}+K^{2}\right)=0, \\
\mathcal{C}_{i} & \equiv D_{j} K^{j}{ }_{i}-D_{i} K=0 .
\end{aligned}
$$

Here an overdot denotes a partial derivative with respect to the time coordinate $(\partial / \partial t)$, indices are raised with the inverse metric $\gamma^{i j}, D_{i}$ is the covariant three-derivative consistent with $\gamma_{i j}, R_{i j}$ is the Ricci curvature tensor of $\gamma_{i j}, R$ its Ricci scalar, $K_{i j}$ is the extrinsic curvature of the slice at fixed value of $t$ and $K \equiv \gamma^{i j} K_{i j}$. Expressed in terms of the Einstein tensor, the constraints (3) are related to specific components in the coordinates $\left(x^{i}, t\right)$ :

$$
\begin{aligned}
\mathcal{C} & =\alpha^{2} G^{t t} \\
\mathcal{C}_{i} & =-\alpha \gamma_{i j} G^{j t},
\end{aligned}
$$

where (4b) holds only for vanishing shift vector. The constraint character (the absence of second derivatives with respect to time) is a consequence of the fact that the only components of the Einstein tensor that appear in (4) have a contravariant index of value $t$. In geometric terms, (4) are linear combinations of $G_{a b} n^{b}=0$ where $n^{b}$ is the unit normal to the slices of fixed value of $t$, and is therefore given by $n^{a}=g^{a b} n_{b}=-\alpha g^{a t}=\delta_{t}^{a} / \alpha$.

Similarly, at any boundary given by a fixed value of a spatial coordinate, the normal vector to the boundary surface $e^{b}$ can be used to project the Einstein tensor as $G_{a b} e^{b}$ in order to obtain the components that have no second derivatives across the boundary. As we have shown previously [2], the vanishing of these can then be imposed as conditions on the boundary values for the fundamental variables. To fix ideas let's choose a boundary surface at a fixed value of $x$. Thus $e^{b}=g^{b x}=\left(0, \gamma^{i x}\right)$ up to scaling. Consequently, $G_{a b} e^{b}=G_{a}^{x}$, so any linear combination of the components of the Einstein tensor with a contravariant index of value $x$ will be suitable. Explicitly we have:

$$
\begin{aligned}
G_{t}^{x}= & -\frac{1}{2} \gamma^{i x}\left((\ln \gamma){ }_{i t}-\gamma^{k l} \dot{\gamma}_{i k, l}\right)-K D^{x} \alpha+K_{k}^{x} D^{k} \alpha \\
& +\alpha\left(\gamma^{k l} \Gamma^{j}{ }_{k l} K_{j}^{x}+\gamma^{i x} \Gamma^{j}{ }_{i k} K_{j}^{k}\right) \\
G_{x}^{x}= & \frac{\dot{K}-\dot{K}_{x}^{x}}{\alpha}-\frac{1}{2}\left(R+K^{i j} K_{i j}+K^{2}\right)+K K_{x}^{x} \\
& +R_{x}^{x}+\frac{1}{\alpha}\left(D^{j} D_{j} \alpha-D^{x} D_{x} \alpha\right) \\
& G_{y}^{x}=-\frac{\dot{K}_{y}^{x}}{\alpha}+K K_{y}^{x}+R_{y}^{x}-\frac{1}{\alpha} D^{x} D_{y} \alpha \\
& G_{z}^{x}=-\frac{\dot{K}_{z}^{x}}{\alpha}+K K_{z}^{x}+R_{z}^{x}-\frac{1}{\alpha} D^{x} D_{z} \alpha
\end{aligned}
$$

Here $\Gamma^{k}{ }_{i j}=(1 / 2) \gamma^{k l}\left(\gamma_{i l, j}+\gamma_{j l, i}-\gamma_{i j, l}\right)$, and the time derivative of the components of the extrinsic curvature is applied after raising an index, that is: $\dot{K}_{j}^{i} \equiv\left(\gamma^{i k} K_{k j}\right)_{,}$. The reader can verify that $R_{y}^{x}$ and $R_{z}^{x}$ do not involve second derivatives with respect to $x$ of any of the variables and that the combination $R_{x}^{x}-\frac{1}{2} R$ doesn't either [1].

Since Eqs. (5) represent the vanishing of four independent components of the Einstein tensor, they must be expressible in terms of linear combinations of the evolution equations (2) and the constraints (3). For this reason, it could mistakenly be thought that they must be satisfied identically by the solution of the evolution equations with initial data satisfying the constraints. Such would be the case only in regions of spacetime where the constraints are satisfied, as well as the evolution equations. Since the constraints are only imposed on the initial data, it is not at all clear that they are satisfied everywhere. A brief calculation that involves taking a time derivative of the constraints and using the evolution equations yields

$$
\begin{aligned}
\dot{\mathcal{C}} & =\alpha \partial^{i} \mathcal{C}_{i}+\ldots \\
\dot{\mathcal{C}}_{i} & =\alpha \partial_{i} \mathcal{C}+\ldots
\end{aligned}
$$

where ... denote undifferentiated terms. This system is strongly hyperbolic 12] because it has real characteristic speeds and a complete set characteristic fields. With respect to the unit vector $\xi^{i}=\gamma^{i x} / \sqrt{\gamma^{x x}}$, normal to the boundary, the constraints $\mathcal{C}^{y}$ and $\mathcal{C}^{z}$ travel with zero characteristic speeds, whereas $\mathcal{C}^{ \pm} \equiv \mathcal{C} \pm \mathcal{C}^{x} / \sqrt{\gamma^{x x}}$ travel with speeds $\pm \alpha$, respectively. Only $\mathcal{C}^{y}, \mathcal{C}^{z}$ and $\mathcal{C}+\mathcal{C}^{x} / \sqrt{\gamma^{x x}}$ are vanishing at the boundary if they are set to zero on the region of the initial slice interior to 
the boundary. Because $\mathcal{C}-\mathcal{C}^{x} / \sqrt{\gamma^{x x}}$ is incoming, it is not vanishing at the boundary be virtue of the evolution and the initial data alone, therefore it is an example of what we refer to, colloquially, as an "un-preserved" constraint, and must be made to vanish at the boundary by an appropriate choice of boundary conditions.

One may not, thus, dismiss the boundary equations (15) as a matter of principle. On the contrary, it is enlightening to see exactly how the boundary equations relate to the constraints.

To start with, by inspection one can clearly see that $G_{y}^{x}$ and $G_{z}^{x}$ are identical to the evolution equations (3) for the mixed components $K_{y}^{x}$ and $K_{z}^{x}$ of the extrinsic curvature (namely: the components $j=y$ and $j=z$ of Eq. (2b) multiplied by $\gamma^{i x}$ ). Thus two of the four boundary equations are identically satisfied by the solution of the evolution equations (irrespective of the initial data). That is not the case with $G_{x}^{x}$. If one uses the evolution equations for $K_{x}^{x}$ and for the trace $K$ that follow from (2b) to eliminate the time derivatives from $G_{x}^{x}$, one is left with the Hamiltonian constraint $\mathcal{C}$. Finally, using the definition of the extrinsic curvature (2a) in the expression for $G_{t}^{x}$ it is straightforward to see that $G_{t}^{x}=\alpha \gamma^{x i} \mathcal{C}_{i}$. Summarizing, if we represent the evolution equations (2a) and (2b) in the form $\mathcal{E}_{i j}^{\gamma}=0$ and $\mathcal{E}_{i j}^{K}=0$ respectively by transferring all the terms from the right into the left-hand side, we have

$$
\begin{aligned}
G_{t}^{x} & =\alpha \mathcal{C}^{x}+\frac{1}{2} \gamma^{x j} \gamma^{k l}\left(\partial_{l} \mathcal{E}_{j k}^{\gamma}-\gamma^{x l} \gamma^{j k} \partial_{l} \mathcal{E}_{j k}^{\gamma}\right) \\
G_{y}^{x} & =\frac{1}{\alpha} \gamma^{x j} \mathcal{E}_{j y}^{K} \\
G_{z}^{x} & =\frac{1}{\alpha} \gamma^{x j} \mathcal{E}_{j z}^{K} \\
G_{x}^{x} & =\mathcal{C}+\frac{1}{\alpha}\left(\gamma^{k l} \mathcal{E}_{k l}^{K}-\gamma^{x j} \mathcal{E}_{x j}^{K}\right)
\end{aligned}
$$

This means that $G_{t}^{x}$ and $G_{x}^{x}$ are equivalent to $\mathcal{C}^{x}$ and $\mathcal{C}$ respectively (modulo the evolution), whereas $G_{y}^{x}$ and $G_{z}^{x}$ are equivalent to zero (modulo the evolution). One can see that imposing either $G_{t}^{x}=0$ or $G_{x}^{x}=0$ is equivalent to a boundary condition of the form $\mathcal{C}^{-}=B \mathcal{C}^{+}$with a constant $B$ on the system of evolution of the constraints. This is a valid boundary condition in the sense that it preserves the well-posedness of the system of evolution of the constraints 12]. Thus, of the two components $G_{t}^{x}=0$ or $G_{x}^{x}=0$, one represents a necessary boundary condition. The other one (or a linear combination of $G_{t}^{x}=0$ and $G_{x}^{x}=0$ not equivalent to the first one) becomes redundant because it represents a condition on the outgoing characteristic field $\mathcal{C}^{+}$, which is already determined at the boundary by the initial values.

Because the ADM evolution equations are not strongly hyperbolic, perhaps nothing else can be said about the role of the equations $G_{a b} e^{b}=0$ as boundary conditions for the ADM equations, except that failure to impose the nontrivial one leads to constraint violations with guaranteed certainty.

In the next Section, we examine the Einstein boundary conditions as applied in the formulation of the Einstein equations that appears in [6].

\section{BOUNDARY CONDITIONS FOR THE BSSN FORMULATION WITH VANISHING SHIFT}

In the case of vanishing shift vector, the formulation of the Einstein equations referred to as BSSN [6] consists of the following evolution equations

$$
\begin{aligned}
\dot{\tilde{\gamma}}_{i j}= & -2 \alpha \tilde{A}_{i j} \\
\dot{\phi}= & -\frac{\alpha}{6} K \\
\dot{\tilde{A}}_{i j}= & \alpha e^{-4 \phi}\left(-\frac{1}{2} \tilde{\gamma}^{l m} \tilde{\gamma}_{i j, l m}+\tilde{\gamma}_{k(i} \tilde{\Gamma}^{k}{ }_{j j}\right)+\tilde{\Gamma}^{k} \tilde{\Gamma}_{(i j) k} \\
& +2 \tilde{\Gamma}^{k l}{ }_{(i} \tilde{\Gamma}_{i) k l}+\tilde{\Gamma}^{k l}{ }_{i} \tilde{\Gamma}_{k l j}-2 \tilde{D}_{i} \tilde{D}_{j} \phi+4 \tilde{D}_{i} \phi \tilde{D}_{j} \phi \\
& -\frac{1}{3} \tilde{\gamma}_{i j}\left(\tilde{\Gamma}^{k},{ }_{k}+\tilde{\Gamma}^{k l i}\left(2 \tilde{\Gamma}_{i k l}+\tilde{\Gamma}_{k l i}\right)\right) \\
& \left.+\frac{2}{3} \tilde{\gamma}_{i j}\left(\tilde{D}^{l} \tilde{D}_{l} \phi-2 \tilde{D}^{l} \phi \tilde{D}_{l} \phi\right)-\frac{\left(D_{i} D_{j} \alpha\right)}{\alpha F}\right) \\
& +K \tilde{A}_{i j}-2 \tilde{A}_{i l} \tilde{A}_{j}^{l} \\
\dot{K}= & \gamma^{i j} D_{i} D_{j} \alpha+\alpha\left(\tilde{A}_{i j} \tilde{A}^{i j}+\frac{1}{3} K^{2}\right) \\
\dot{\tilde{\Gamma}}^{i}= & 2 \alpha\left(\tilde{\Gamma}^{i}{ }_{j k} \tilde{A}^{k j}-\frac{2}{3} \tilde{\gamma}^{i j} K,_{j}+6 \tilde{A}^{i j} \phi,_{j}\right) \\
& -2 \tilde{A}^{i j} \alpha,_{j}
\end{aligned}
$$

for the 15 variables

$$
\begin{aligned}
\phi & \equiv \frac{1}{12} \ln \left(\operatorname{det} \gamma_{i j}\right) \\
\tilde{\gamma}_{i j} & \equiv e^{-4 \phi} \gamma_{i j} \\
K & \equiv \gamma^{i j} K_{i j} \\
\tilde{A}_{i j} & \equiv e^{-4 \phi}\left(K_{i j}-\frac{1}{3} \tilde{\gamma}_{i j} K\right) \\
\tilde{\Gamma}^{i} & \equiv-\tilde{\gamma}^{i j},_{j}
\end{aligned}
$$

which mainly separate out the determinant of the threemetric and the trace of the extrinsic curvature in order to evolve them as fundamental variables in their own right. But additionally, three first order variables are introduced, $\tilde{\Gamma}^{i}$, corresponding to certain combinations of first derivatives of the metric. This has the effect of modifying the principal symbol of the system of PDE's.

For this system, we will express the boundary equations (5) directly in terms of the fundamental variables, in such a way that no second $x$-derivatives of $\tilde{\gamma}_{i j}$ or $\phi$ occur, nor first $x$-derivatives of $\tilde{\Gamma}^{i}, \tilde{A}_{i j}$ nor $K$ occur.

We start with the simplest one, that is $G_{y}^{x}$, which contains second derivatives in the term $R_{y}^{x}$.

To start with, we have $R_{i j}=\tilde{R}_{i j}+R_{i j}^{\phi}$ where $\tilde{R}_{i j}$ is the Ricci tensor of $\tilde{\gamma}_{i j}$ and

$$
\begin{aligned}
R_{i j}^{\phi}= & -2 \tilde{D}_{i} \tilde{D}_{j} \phi-2 \tilde{\gamma}_{i j} \tilde{D}^{l} \tilde{D}_{l} \phi \\
& +4 \tilde{D}_{i} \phi \tilde{D}_{j} \phi-4 \tilde{\gamma}_{i j} \tilde{D}^{l} \phi \tilde{D}_{l} \phi
\end{aligned}
$$


So $R_{y}^{x}=\gamma^{x j} R_{y j}=e^{-4 \phi} \tilde{\gamma}^{x j}\left(\tilde{R}_{y j}+R_{y j}^{\phi}\right)=e^{-4 \phi}\left(\tilde{R}_{y}^{x}+\right.$ $\left.\tilde{\gamma}^{x j} R_{y j}^{\phi}\right)$. As far as the principal terms are concerned, we have

$$
\tilde{\gamma}^{x j} R_{y j}^{\phi}=-2 \tilde{D}^{x} \tilde{D}_{y} \phi+\ldots
$$

Also as far as principal terms are concerned, the Ricci tensor of a metric of unit determinant is

$$
\tilde{R}_{i j}=-\frac{1}{2} \tilde{\gamma}^{k l}\left(\tilde{\gamma}_{i j, k l}-\tilde{\gamma}_{i l, j k}-\tilde{\gamma}_{j k, i l}\right)+\ldots
$$

Thus

$$
\begin{aligned}
\tilde{R}_{y}^{x}= & -\frac{1}{2} \tilde{\gamma}^{x j} \tilde{\gamma}^{k l}\left(\tilde{\gamma}_{y j, k l}-\tilde{\gamma}_{y l, j k}-\tilde{\gamma}_{j k, y l}\right)+\ldots \\
= & -\frac{1}{2}\left(\tilde{\gamma}^{x m} \tilde{\gamma}^{k l}-\tilde{\gamma}^{x l} \tilde{\gamma}^{k m}\right) \tilde{\gamma}_{y m, k l} \\
& +\frac{1}{2} \tilde{\gamma}^{x m} \tilde{\gamma}^{k l} \tilde{\gamma}_{k m, y l}+\ldots
\end{aligned}
$$

The last term can be substituted in terms of the firstorder variable $\tilde{\Gamma}^{i}$ :

$$
\frac{1}{2} \tilde{\gamma}^{x m} \tilde{\gamma}^{k l} \tilde{\gamma}_{k m, y l}=\frac{1}{2} \tilde{\Gamma}^{x},{ }_{y}+\ldots
$$

Additionally, by expanding the three values of the index $k$ in the first term one can see that $k=x$ vanishes identically, so that the first term contains no second $x$-derivatives of $\tilde{\gamma}_{i j}$ as it stands, and we have explicitly

$$
\begin{aligned}
\tilde{R}_{y}^{x}= & \frac{1}{2}\left(\tilde{\gamma}^{x l} \tilde{\gamma}^{y m}-\tilde{\gamma}^{x m} \tilde{\gamma}^{y l}\right) \tilde{\gamma}_{y m, l y} \\
& +\frac{1}{2}\left(\tilde{\gamma}^{x l} \tilde{\gamma}^{z m}-\tilde{\gamma}^{x m} \tilde{\gamma}^{z l}\right) \tilde{\gamma}_{y m, l z} \\
& +\frac{1}{2} \tilde{\Gamma}^{x},{ }_{y}+\ldots
\end{aligned}
$$

On the other hand, $K_{y}^{x}=\gamma^{x m} K_{m y}=\tilde{\gamma}^{x m}\left(\tilde{A}_{m y}+\right.$ $\left.\frac{1}{3} \tilde{\gamma}_{y m} K\right)=\tilde{A}_{y}^{x}$, so that

$$
\dot{K}_{y}^{x}=\dot{\tilde{A}}_{y}^{x}+\ldots
$$

Finally, thus, the principal terms of $G_{y}^{x}$ are written out in the intended form:

$$
\begin{aligned}
G_{y}^{x}= & -\frac{\dot{\tilde{A}}_{y}^{x}}{\alpha}+e^{-4 \phi}\left(\frac{1}{2}\left(\tilde{\gamma}^{x l} \tilde{\gamma}^{y m}-\tilde{\gamma}^{x m} \tilde{\gamma}^{y l}\right) \tilde{\gamma}_{y m, l y}\right. \\
& +\frac{1}{2}\left(\tilde{\gamma}^{x l} \tilde{\gamma}^{z m}-\tilde{\gamma}^{x m} \tilde{\gamma}^{z l}\right) \tilde{\gamma}_{y m, l z}+\frac{1}{2} \tilde{\Gamma}^{x}, y \\
& \left.-2 \tilde{D}^{x} \tilde{D}_{y} \phi\right)+\ldots
\end{aligned}
$$

By a completely analogous calculation we also have

$$
\begin{aligned}
G_{z}^{x}= & -\frac{\dot{\tilde{A}}_{z}^{x}}{\alpha}+e^{-4 \phi}\left(\frac{1}{2}\left(\tilde{\gamma}^{x l} \tilde{\gamma}^{y m}-\tilde{\gamma}^{x m} \tilde{\gamma}^{y l}\right) \tilde{\gamma}_{z m, l y}\right. \\
& +\frac{1}{2}\left(\tilde{\gamma}^{x l} \tilde{\gamma}^{z m}-\tilde{\gamma}^{x m} \tilde{\gamma}^{z l}\right) \tilde{\gamma}_{z m, l z}+\frac{1}{2} \tilde{\Gamma}^{x}, z \\
& \left.-2 \tilde{D}^{x} \tilde{D}_{z} \phi\right)+\ldots
\end{aligned}
$$

We can treat $G_{x}^{x}$ with a similar procedure. By analogy with (16) the reader should have no trouble to see that

$$
\begin{aligned}
\tilde{R}_{x}^{x}= & \frac{1}{2}\left(\tilde{\gamma}^{x l} \tilde{\gamma}^{y m}-\tilde{\gamma}^{x m} \tilde{\gamma}^{y l}\right) \tilde{\gamma}_{x m, l y} \\
& +\frac{1}{2}\left(\tilde{\gamma}^{x l} \tilde{\gamma}^{z m}-\tilde{\gamma}^{x m} \tilde{\gamma}^{z l}\right) \tilde{\gamma}_{x m, l z} \\
& +\frac{1}{2} \tilde{\Gamma}^{x},{ }_{x}+\ldots
\end{aligned}
$$

But since

$$
\begin{aligned}
\tilde{R} & =\tilde{\gamma}^{i j} \tilde{R}_{i j}=\tilde{\gamma}^{i j} \tilde{\gamma}^{k l} \tilde{\gamma}_{i l, k j}+\ldots \\
& =-\tilde{\gamma}^{k j}, k j+\ldots \\
& =\tilde{\Gamma}^{k}, k+\ldots
\end{aligned}
$$

then the term with an $x$-derivative of $\tilde{\Gamma}^{x}$ in $\tilde{R}_{x}^{x}$ cancels out with a term in $-\frac{1}{2} \tilde{R}$, so that:

$$
\begin{aligned}
\tilde{R}_{x}^{x}-\frac{1}{2} \tilde{R}= & \frac{1}{2}\left(\tilde{\gamma}^{x l} \tilde{\gamma}^{y m}-\tilde{\gamma}^{x m} \tilde{\gamma}^{y l}\right) \tilde{\gamma}_{x m, l y} \\
& +\frac{1}{2}\left(\tilde{\gamma}^{x l} \tilde{\gamma}^{z m}-\tilde{\gamma}^{x m} \tilde{\gamma}^{z l}\right) \tilde{\gamma}_{x m, l z} \\
& -\frac{1}{2} \tilde{\Gamma}^{y}, y-\frac{1}{2} \tilde{\Gamma}^{z}{ }_{z}+\ldots
\end{aligned}
$$

Additionally, we have $K_{x}^{x}=\tilde{A}_{x}^{x}+\frac{1}{3} K$, so that

$$
\dot{K}_{x}^{x}=\dot{\tilde{A}}_{x}^{x}+\frac{1}{3} \dot{K}+\ldots
$$

Collecting the relevant results, the principal terms of $G_{x}^{x}$ are finaly written out in the intended form:

$$
\begin{aligned}
G_{x}^{x}= & -\frac{\dot{\tilde{A}}_{x}^{x}}{\alpha}+\frac{2}{3 \alpha} \dot{K}+e^{-4 \phi}\left(\frac{1}{2}\left(\tilde{\gamma}^{x l} \tilde{\gamma}^{y m}-\tilde{\gamma}^{x m} \tilde{\gamma}^{y l}\right) \tilde{\gamma}_{x m, l y}\right. \\
& +\frac{1}{2}\left(\tilde{\gamma}^{x l} \tilde{\gamma}^{z m}-\tilde{\gamma}^{x m} \tilde{\gamma}^{z l}\right) \tilde{\gamma}_{x m, l z}-\frac{1}{2} \tilde{\Gamma}^{y},{ }_{y}-\frac{1}{2} \tilde{\Gamma}^{z}, z \\
& \left.+2 \tilde{D}^{y} \tilde{D}_{y} \phi+2 \tilde{D}^{z} \tilde{D}_{z} \phi\right)+\ldots
\end{aligned}
$$

For the remaining boundary equation, $G_{t}^{x}$, we observe that

$$
G_{t}^{x}=-\frac{1}{2} \gamma^{x i}\left(\left(\ln \left(\operatorname{det} \gamma_{k l}\right)\right),{ }_{i t}-\gamma^{k l} \dot{\gamma}_{i k, l}\right)+\ldots
$$

which, with the simple substitution of $\gamma_{i j}=e^{4 \phi} \tilde{\gamma}_{i j}$, becomes

$$
G_{t}^{x}=-\frac{1}{2} e^{-4 \phi}\left(8 \tilde{\gamma}^{x i} \dot{\phi},_{i}+\dot{\gamma}^{x l}, l\right)+\ldots
$$

which we take as the intended form without further substitutions.

In principle, thus, we have four boundary equations

$$
\begin{aligned}
G_{t}^{x} & =0, \\
G_{x}^{x} & =0, \\
G_{y}^{x} & =0, \\
G_{z}^{x} & =0,
\end{aligned}
$$


with $G_{y}^{x}, G_{z}^{x}, G_{x}^{x}, G_{t}^{x}$ given by (18), (19), (24) and (26), as candidates for nontrivial boundary conditions for the evolution equations (8). Notice that, unlike the ADM case, none of the four equations is manifestly identical to any of the evolution equations (8), the reason being that all the evolution equations for the traceless extrinsic curvature density $\tilde{A}_{i j}$ contain second derivatives of $\tilde{\gamma}_{i j}$ as part of the Laplacian operator $\tilde{\gamma}^{k l} \partial_{k} \partial_{l}$. This makes it a nontrivial task to figure out whether the boundary equations are identically satisfied by the solution of the evolution equations with constrained initial data. In the following Section, we argue that at least three of them are necessary, on the basis of their role in ensuring the propagation of the vanishing values of the constraints.

\section{RELATION TO CONSTRAINT PROPAGATION}

The scalar and vector constraints $\mathcal{C}$ and $\mathcal{C}_{i}$ of (3) can be expressed as follows in terms of the BSSN variables:

$$
\begin{aligned}
\mathcal{C} \equiv & e^{-4 \phi}\left(\tilde{\Gamma}_{, k}^{k}-8 \tilde{D}^{l} \tilde{D}_{l} \phi-8 \tilde{D}^{l} \phi \tilde{D}_{l} \phi\right. \\
& \left.+\frac{1}{4} \tilde{\gamma}^{l m} \tilde{\gamma}^{j k}, l\left(\tilde{\gamma}_{j k, m}-2 \tilde{\gamma}_{j m, k}\right)\right) \\
& -\tilde{A}_{i j} \tilde{A}^{i j}-\frac{2}{3} K^{2}, \\
\mathcal{C}_{i} \equiv & \tilde{\gamma}^{j k} \tilde{A}_{k i, j}-\tilde{\Gamma}^{k} \tilde{A}_{i k}-\frac{1}{2} \tilde{\gamma}_{j k, i} \tilde{A}^{j k}-\frac{2}{3} K,_{i} \\
& +6 \tilde{A}_{i}^{j} \phi, j
\end{aligned}
$$

In addition to the scalar and vector constraints $\mathcal{C}$ and $\mathcal{C}_{i}$, an additional constraint,

$$
\mathcal{G}^{i} \equiv \tilde{\Gamma}^{i}+\tilde{\gamma}^{i j}{ }_{j}
$$

must be imposed on the initial values, i.e.,

$$
\mathcal{G}^{i}=0
$$

in order for the BSSN equations (8) to yield a solution of the ADM equations (2). Whether the constraints $\mathcal{C}, \mathcal{C}_{i}$ and $\mathcal{G}^{i}$ are satisfied by the solution of the evolution equations (8) is determined by the equations for $\dot{\mathcal{C}}, \dot{\mathcal{C}}_{i}$ and $\dot{\mathcal{G}}^{i}$ that are implied by (8). The easiest propagation equation to obtain is that for $\mathcal{G}^{i}$, because $\mathcal{G}^{i}$ is a trivial constraint, whose propagation should be exactly zero by construction unless some constraint had been used in establishing the evolution equations for $\tilde{\Gamma}^{i}$ or for $\tilde{\gamma}_{i j}$. Since the vector constraint is necessary to write Eq. (8e) in such a form, the evolution of $\mathcal{G}^{i}$ naturally depends on the vector constraint, as follows:

$$
\dot{\mathcal{G}}^{i}=2 \alpha \tilde{\gamma}^{i j} \mathcal{C}_{j}
$$

This equation has no principal terms for the purposes of the structure of characteristics, so we can write it as

$$
\dot{\mathcal{G}}^{i}=\ldots
$$

where ... represents undifferentiated terms. Additionally, since the time-derivatives of the variables that appear in the expression (28a) for $\mathcal{C}$ will not contribute any terms that would have derivatives of the constraints themselves, we have

$$
\dot{\mathcal{C}}=\ldots
$$

as well. Finally, the only term that contributes derivatives of constraints to $\dot{\mathcal{C}}_{i}$ is $\tilde{\gamma}^{j k} \dot{\tilde{A}}_{k i, j}$, yielding

$$
\dot{\mathcal{C}}_{i}=\frac{\alpha e^{-4 \phi}}{2} \tilde{\gamma}_{i k} \tilde{D}^{l} \tilde{D}_{l} \mathcal{G}^{k}+\frac{\alpha}{6} \mathcal{C},_{i}+\ldots
$$

As it stands, the system of evolution equations (31), (32) and (33) is not manifestly well posed, not even manifestly hyperbolic, because of the presence of second space derivatives in the right-hand side as a direct consequence of the addition of the three first-order variables $\tilde{\Gamma}^{i}$. In order to facilitate the study of the properties of propagation of the constraints, however, we may define a set of six first-order variables for the constraints that allow us to reduce the differential order of the right-hand side:

$$
\mathcal{I}_{i k} \equiv \tilde{\gamma}_{i l} \mathcal{G}^{l}, k
$$

These are not to be thought of as additional constraints to be imposed on the initial data of the BSSN evolution equations. Rather, they will be automatically satisfied by any set of initial data that satisfies $\mathcal{G}^{i}=0$. But they are useful because, by adding them as new variables, the propagation of the constraints takes the form:

$$
\begin{aligned}
\dot{\mathcal{C}} & =\ldots \\
\dot{\mathcal{C}}_{i} & =\frac{\alpha}{6} \mathcal{C},_{i}+\frac{\alpha e^{-4 \phi}}{2} \tilde{\gamma}^{k l} \mathcal{I}_{i k, l}+\ldots \\
\dot{\mathcal{I}}_{i k} & =2 \alpha \mathcal{C}_{i, k}+\ldots \\
\dot{\mathcal{G}}^{i} & =\ldots
\end{aligned}
$$

This first-order system of 13 equations is strongly hyperbolic in the sense that it has a complete set of null eigenvectors. This means that for each arbitrary covector $\xi_{i}$ of unit length $\xi_{i} \xi_{j} \gamma^{i j}=1$ at any given point, there are 13 characteristic fields, which travel with real speeds. One can easily see that the characteristic speeds of this system are 0 , and $\pm \alpha$, with multiplicity 7,3 and 3 respectively. Thus seven characteristic constraints are "static" in the sense that they propagate along the direction of evolution $\partial / \partial t$. Of the six non-static characteristic constraints, three are outgoing at the boundary:

$$
{ }^{+} \mathcal{Z}_{i} \equiv \mathcal{C}_{i}+\frac{1}{2} \xi^{k} \mathcal{I}_{i k}+\frac{1}{6} \xi_{i} \mathcal{C}
$$

and three are incoming:

$$
{ }^{-} \mathcal{Z}_{i} \equiv \mathcal{C}_{i}-\frac{1}{2} \xi^{k} \mathcal{I}_{i k}-\frac{1}{6} \xi_{i} \mathcal{C}
$$

This indicates that, even if the 13 constraints are set to zero on the initial slice, they would not remain zero in 
the future of the initial slice unless the incoming constraints are set to zero, either directly or indirectly as functions of the outgoing constraints. One way to accomplish this would be by means of boundary conditions of the form ${ }^{-} \mathcal{Z}_{i}=L_{i j}{ }^{+} \mathcal{Z}_{j}$ with constant coefficients $L_{i j}$, which is a necessary condition in order to preserve the well-posedness of the propagation of the constraints [12].

Our next goal is to show that the boundary equations $G_{a}^{x}=0$ with $G_{a}^{x}$ given by (18), (19), (24) and (26) are equivalent, in a sense, to such boundary conditions. This would indicate, in effect, that the boundary equations $G_{a}^{x}=0$ are instrumental in preserving the constraints.

More precisely, we wish to show that the boundary equations $G_{a}^{x}=0$ are related to the non-static constraints through linear combinations with the evolution equations in the following manner:

$$
\begin{aligned}
G_{t}^{x} & =-\alpha \mathcal{C}^{x}-\frac{1}{2} \gamma^{x l}\left(8 \tilde{\mathcal{E}}^{\phi}{ }_{l}-\tilde{\gamma}^{k j} \tilde{\mathcal{E}}_{l k, j}^{\gamma}\right) \\
G_{x}^{x} & =-\left(\frac{1}{6} \mathcal{C}+\frac{1}{2} \xi^{m} \mathcal{I}_{x m}\right)-\frac{1}{\alpha} \tilde{\gamma}^{x j} \tilde{\mathcal{E}}_{x j}^{A}+\frac{2}{3 \alpha} \tilde{\mathcal{E}}^{K} \\
G_{y}^{x} & =-\frac{1}{2} \xi^{m} \mathcal{I}_{y m}-\frac{1}{\alpha} \gamma^{x j} \tilde{\mathcal{E}}_{y j}^{A} \\
G_{z}^{x} & =-\frac{1}{2} \xi^{m} \mathcal{I}_{z m}-\frac{1}{\alpha} \gamma^{x j} \tilde{\mathcal{E}}_{z j}^{A}
\end{aligned}
$$

where $\tilde{\mathcal{E}}^{\phi}, \tilde{\mathcal{E}}_{l k}^{\gamma}, \tilde{\mathcal{E}}_{i j}^{A}, \tilde{\mathcal{E}}^{K}$ represent the evolution equations (8b), 8a , (8c) and (8d) where all the terms in the righthand side are moved to the left. We can think of the relationship between the boundary equations and the constraints through the evolution equations as an equivalence:

$$
\begin{aligned}
G_{t}^{x} & \sim-\alpha \mathcal{C}^{x} \\
G_{x}^{x} & \sim-\left(\frac{1}{6} \mathcal{C}+\frac{1}{2} \xi^{m} \mathcal{I}_{x m}\right) \\
G_{y}^{x} & \sim-\frac{1}{2} \xi^{m} \mathcal{I}_{y m} \\
G_{z}^{x} & \sim-\frac{1}{2} \xi^{m} \mathcal{I}_{z m}
\end{aligned}
$$

modulo the evolution.

In order to prove this, we start with the boundary equation $G_{x}^{x}=0$. We have, explicitly from (8c),

$$
\begin{aligned}
\tilde{\gamma}^{x l} \tilde{\mathcal{E}}_{x l}^{A}= & \dot{\tilde{A}}_{x}^{x}-\alpha e^{-4 \phi}\left(-\frac{1}{2} \tilde{\gamma}^{k l} \tilde{\gamma}^{x j} \tilde{\gamma}_{x j, k l}+\frac{1}{2} \tilde{\Gamma}^{x}{ }_{, x}\right. \\
& \left.+\frac{1}{2} \tilde{\gamma}^{x j} \tilde{\gamma}_{k x} \tilde{\Gamma}^{k}{ }_{j}-\frac{1}{3} \tilde{\Gamma}^{k}{ }_{, k}-2 \tilde{D}^{x} \tilde{D}_{x} \phi+\frac{2}{3} \tilde{D}^{k} \tilde{D}_{k} \phi\right) \\
& +\ldots
\end{aligned}
$$

and also

$$
\tilde{\gamma}^{x l} \tilde{\mathcal{E}}_{x l}^{K}=\dot{K}+\ldots
$$

Therefore, adding the evolution equations (with appropriate factors) to $G_{x}^{x}$ as given by Eq. (24), we have immediately

$$
\begin{aligned}
G_{x}^{x}+ & \frac{1}{\alpha} \tilde{\gamma}^{x j} \tilde{\mathcal{E}}_{x j}^{A}-\frac{2}{3 \alpha} \tilde{\mathcal{E}}^{K} \\
= & \frac{e^{-4 \phi}}{2}\left(\tilde{\gamma}^{x j} \tilde{\gamma}^{k l} \tilde{\gamma}_{x j, k l}+\left(\tilde{\gamma}^{x l} \tilde{\gamma}^{y m}-\tilde{\gamma}^{x m} \tilde{\gamma}^{y l}\right) \tilde{\gamma}_{x m, l y}\right. \\
& +\left(\tilde{\gamma}^{x l} \tilde{\gamma}^{z m}-\tilde{\gamma}^{x m} \tilde{\gamma}^{z l}\right) \tilde{\gamma}_{x m, l z}-\frac{1}{3} \tilde{\Gamma}^{k}{ }_{, k}+\frac{8}{3} \tilde{D}^{k} \tilde{D}_{k} \phi \\
& \left.-\tilde{\gamma}^{x j} \tilde{\gamma}_{k x} \tilde{\Gamma}^{k},{ }_{j}\right)+\ldots
\end{aligned}
$$

The three indicated terms in second derivatives of $\tilde{\gamma}_{i j}$ manifestly combine to yield

$$
\begin{aligned}
&\left(\tilde{\gamma}^{x l} \tilde{\gamma}^{y m}-\tilde{\gamma}^{x m} \tilde{\gamma}^{y l}\right) \tilde{\gamma}_{x m, l y} \\
&+\left(\tilde{\gamma}^{x l} \tilde{\gamma}^{z m}-\tilde{\gamma}^{x m} \tilde{\gamma}^{z l}\right) \tilde{\gamma}_{x m, l z} \\
&+
\end{aligned}
$$

Thus (42) reads, equivalently,

$$
\begin{aligned}
G_{x}^{x}+\frac{1}{\alpha} \tilde{\gamma}^{x j} \tilde{\mathcal{E}}_{x j}^{A}-\frac{2}{3 \alpha} \tilde{\mathcal{E}}^{K}= & \left(\tilde{\gamma}^{x m}\left(\tilde{\gamma}^{k l} \tilde{\gamma}_{x l, k m}-\tilde{\gamma}_{k x} \tilde{\Gamma}^{k}{ }_{, m}\right)\right. \\
& \left.-\frac{1}{3}\left(\tilde{\Gamma}^{k}{ }_{, k}-8 \tilde{D}^{l} \tilde{D} l \phi\right)\right) \frac{e^{-4 \phi}}{2} \\
& +\ldots
\end{aligned}
$$

Both indicated terms are directly expressible in terms of constraints now. The second term in parenthesis (with the exponential factor included) is manifestly equal to the Hamiltonian constraint $\mathcal{C}$, whereas the first parenthesis immediately leads to one of the new first-order constraints:

$$
\begin{aligned}
\tilde{\gamma}^{k l} \tilde{\gamma}_{x l, k m}-\tilde{\gamma}_{k x} \tilde{\Gamma}^{k}{ }_{, m} & =-\tilde{\gamma}_{k x}\left(\tilde{\Gamma}^{k}+\tilde{\gamma}^{k j},_{j}\right){ }_{, m}+\ldots \\
& =-\tilde{\gamma}_{k x} \mathcal{G}^{k},_{m}+\ldots \\
& =-\mathcal{I}_{x m}+\ldots
\end{aligned}
$$

Thus Eq. 38b is verified.

We now show how to prove (38c). From (8c) we have

$$
\begin{aligned}
\frac{1}{\alpha} \gamma^{x j} \tilde{\mathcal{E}}_{y j}^{A}= & \frac{e^{-4 \phi}}{2}\left(\tilde{\gamma}^{x j} \tilde{\gamma}^{l m} \tilde{\gamma}_{y j, l m}-\tilde{\gamma}^{x j} \tilde{\gamma}_{y k} \tilde{\Gamma}^{k}{ }_{j}-\tilde{\Gamma}^{x},{ }_{y}\right. \\
& \left.+4 \tilde{D}^{x} \tilde{D}_{y} \phi\right)+\dot{\tilde{A}}_{y}^{x}+\ldots
\end{aligned}
$$

Adding this to $G_{y}^{x}$ as given by (18) we manifestly obtain

$$
\begin{aligned}
G_{y}^{x}+\frac{1}{\alpha} \gamma^{x j} \tilde{\mathcal{E}}_{y j}^{A}= & \left(\left(\tilde{\gamma}^{x l} \tilde{\gamma}^{y m}-\tilde{\gamma}^{x m} \tilde{\gamma}^{y l}\right) \tilde{\gamma}_{y m, l y}\right. \\
& +\left(\tilde{\gamma}^{x l} \tilde{\gamma}^{z m}-\tilde{\gamma}^{x m} \tilde{\gamma}^{z l}\right) \tilde{\gamma}_{y m, l z} \\
& \left.+\tilde{\gamma}^{x j} \tilde{\gamma}^{l m} \tilde{\gamma}_{y j, l m}-\tilde{\gamma}^{x j} \tilde{\gamma}_{y k} \tilde{\Gamma}^{k}, j\right) \frac{e^{-4 \phi}}{2} \\
& +\ldots
\end{aligned}
$$

By expanding the index $l=x, y, z$ in the third line of the preceeding equation some cancellations take place, leading directly to

$G_{y}^{x}+\frac{1}{\alpha} \gamma^{x j} \tilde{\mathcal{E}}_{y j}^{A}=\frac{e^{-4 \phi}}{2}\left(\tilde{\gamma}^{x l} \tilde{\gamma}^{k m} \tilde{\gamma}_{y m, k l}-\tilde{\gamma}^{x j} \tilde{\gamma}_{y k} \tilde{\Gamma}^{k}{ }_{j}\right)+\ldots$ 
The terms in parenthesis in the right-hand side are now expressible in terms of the new first-order constraint:

$$
\begin{aligned}
\tilde{\gamma}^{x l} \tilde{\gamma}^{k m} \tilde{\gamma}_{y m, k l}-\tilde{\gamma}^{x j} \tilde{\gamma}_{y k} \tilde{\Gamma}^{k}{ }_{, j} & =-\tilde{\gamma}^{x j} \tilde{\gamma}_{y k}\left(\tilde{\gamma}^{k l},{ }_{l}+\tilde{\Gamma}^{k}\right),{ }_{j}+\ldots \\
& =-\tilde{\gamma}^{x j} \mathcal{I}_{y j}+\ldots
\end{aligned}
$$

Thus Eq. (38c) is verified. A completely analogous procedure (with $z$ in the place of the subscript $y$ ) leads to the verification of Eq. (38d).

It only remains to verify Eq. (38a). From (8b) and (8a) we have trivially

$$
\tilde{\mathcal{E}}^{\phi}=\dot{\phi}+\ldots
$$

and

$$
\tilde{\mathcal{E}}_{l k}^{\gamma}=\dot{\tilde{\gamma}}_{l k}+\ldots
$$

Adding these (in the manner specified by (38a)) to $G_{t}^{x}$ as given by (26) we have immediately

$$
\begin{aligned}
& G_{t}^{x}+\frac{1}{2} \gamma^{x l}\left(8 \tilde{\mathcal{E}}^{\phi}{ }_{l}-\tilde{\gamma}^{k j} \tilde{\mathcal{E}}_{l k, j}^{\gamma}\right) \\
& =e^{-4 \phi} \alpha\left(\frac{2}{3} \tilde{\gamma}^{x l} K_{, l}-\tilde{\gamma}^{x l} \tilde{\gamma}^{k j} \tilde{A}_{k l, j}\right)+\ldots
\end{aligned}
$$

The right-hand side of the preceeding equation is manifestly expressible in terms of the vector constraint $\mathcal{C}_{l}$, with the consequence that Eq. (38a is verified. By the set of equations (38), thus, imposing the equations $G_{a}^{x}=0$ on the boundary is equivalent, in a sense, to imposing the indicated combinations of constraints on the boundary. We now show that such combinations of constraints are equivalent to the incoming characteristic constraints.

By Eqs. (38), (36) and (37), using $\xi_{i}=(1,0,0) / \sqrt{\gamma^{x x}}$, we have

$$
\begin{aligned}
G_{t}^{x} & \sim-\frac{\alpha}{2} \gamma^{x i}\left({ }^{-} \mathcal{Z}_{i}+{ }^{+} \mathcal{Z}_{i}\right) \\
G_{x}^{x} & \sim \frac{1}{2}\left({ }^{-} \mathcal{Z}_{x}-{ }^{+} \mathcal{Z}_{x}\right) \\
G_{y}^{x} & \sim \frac{1}{2}\left({ }^{-} \mathcal{Z}_{y}-{ }^{+} \mathcal{Z}_{y}\right) \\
G_{z}^{x} & \sim \frac{1}{2}\left({ }^{-} \mathcal{Z}_{z}-{ }^{+} \mathcal{Z}_{z}\right)
\end{aligned}
$$

The four components $G_{a}^{x}$ are equivalent (modulo the evolution) to linear combinations of the incoming and outgoing constraints with constant coefficients. Thus imposing $G_{a}^{x}=0$ is equivalent (modulo evolution) to setting wellposed boundary conditions on the system of propagation of the constraints $\left({ }^{-} \mathcal{Z}_{i}=L_{i}^{j}+\mathcal{Z}_{j}\right)$. The propagation of the constraints requires only three boundary conditions. Accordingly, three linearly independent combinations of the four equations $G_{a}^{x}=0$ are necessary in order to ensure that the constraints will remain satisfied by the solution of the evolution equations (8) with constrained initial data. A fourth lindearly independent one should be satisfied identically.
This means that by imposing three (linearly independent combinations) of the four equations $G_{a}^{x}=0$ on the boundary values of the evolution equations (8) the propagation of the constraints is enforced.

At this level there is no preferred set of three linear combinations out of the four equations $G_{a}^{x}=0$. This freedom is an advantage, because it may easily accomodate requirements that may stem from expectations of numerical stability. We do not concern ourselves with such considerations in this work.

The question arises as to whether the fourth equation should be used on the boundary values. For the sake of argument, we can take the fourth equation to be $G_{t}^{x}+\alpha \gamma^{x i} G_{i}^{x}=0$, since this equation is equivalent to a linear combination of purely outgoing constraints $\left(+\mathcal{Z}_{i} \gamma^{x i}=0\right)$. If one looks at this question from the point of view of the constraint-propagation problem (35), clearly imposing the fourth equation would be redundant, given that the outgoing constraints are made to vanish at the boundary simply by picking vanishing initial values. Yet the fourth equation is not inconsistent with the choice of vanishing initial values for the outgoing constraints.

From the point of view of the evolution equations (8), however, it is not at all clear that the fourth equation should not be imposed. This is because, as it stands, the system of evolution equations (8) is not strongly hyperbolic in the standard sense (see, however, 13]). Therefore, there is no clear classification of the 15 fundamental variables into static, incoming or outgoing fields. As a consequence, to our knowledge, the actual number of boundary conditions required by the evolution equations (8), being not less than three, remains unknown.

In the next section we reduce the BSSN equations fully to first-order form. The resulting first-order system is strongly hyperbolic in the standard sense and the boundary-value-problem can be solved in the standard manner.

\section{EINSTEIN BOUNDARY CONDITIONS FOR A WELL-POSED FIRST-ORDER REDUCTION OF THE BSSN EQUATIONS}

In order for Eqs. (8) to reduce to first-order form, the spatial derivatives of $\phi$ and those of $\tilde{\gamma}_{i j}$ not already accounted for by $\tilde{\Gamma}^{i}$ need to be added as new variables (a total of 15 new variables). They are

$$
\begin{aligned}
Q_{i} & \equiv \phi_{i} \\
V_{i j k} & \equiv \tilde{\gamma}_{i j, k}-\frac{3}{5} \tilde{\gamma}_{k(i} \tilde{\gamma}_{j) l, m} \tilde{\gamma}^{l m}+\frac{1}{5} \tilde{\gamma}_{i j} \tilde{\gamma}_{k l, m} \tilde{\gamma}^{l m}(55 \mathrm{~b})
\end{aligned}
$$

These new first-order variables have been used before in order to find a symmetrizable hyperbolic first-order reduction of the BSSN equations by linear combination with the constraints 14]. The difference here is that we do not involve combinations with the constraints. By 
using $\tilde{\gamma}^{i j}$ to raise the first two indices in $55 \mathrm{~b}$ we have

$$
V^{i j}{ }_{k}=-\tilde{\gamma}^{i j}{ }_{k}+\frac{3}{5} \delta_{k}^{(i} \tilde{\gamma}^{j) m}{ }_{m}-\frac{1}{5} \tilde{\gamma}^{i j} \tilde{\gamma}^{l m}{ }_{m} \tilde{\gamma}_{k l}
$$

One has $V^{i j}{ }_{j}=0$ automatically, so the set of variables $V^{i j}{ }_{k}$ does not include the three variables $\tilde{\Gamma}^{i}$. Additionally, $V^{i j}{ }_{k}$ is traceless in the first two indices, also by construction $\left(V^{i j}{ }_{k} \tilde{\gamma}_{i j}=0\right)$. Thus $V^{i j}{ }_{k}$ are 12 variables in all. Using $V^{i j}{ }_{k}$ and $\tilde{\Gamma}^{i}$ one is able to invert in order to obtain all the derivatives of the inverse metric in terms of the new variables:

$$
\tilde{\gamma}^{i j}{ }_{k}=-V_{k}^{i j}-\frac{3}{5} \delta_{k}^{(i} \tilde{\Gamma}^{j)}+\frac{1}{5} \tilde{\gamma}^{i j} \tilde{\Gamma}^{l} \tilde{\gamma}_{k l}
$$

In addition to reducing the BSSN equations to firstorder form, we also require the lapse function to be densitized, namely:

$$
\alpha=\sigma \sqrt{\operatorname{det}\left(\gamma_{i j}\right)}=\sigma e^{6 \phi}
$$

where $\sigma$ is an arbitrarily prescribed function of spacetime. The evolution equations (8), augmented by trivial evolution equations for $Q_{i}$ and $V^{i j}{ }_{k}$, read now:

$$
\begin{aligned}
\dot{\tilde{\gamma}}_{i j}= & -2 \alpha \tilde{A}_{i j} \\
\dot{\phi}= & -\frac{\alpha}{6} K \\
\dot{\tilde{A}}_{i j}= & -\left(\frac{1}{2} \tilde{\gamma}_{i r} \tilde{\gamma}_{j s} \tilde{\gamma}^{k m} V_{k, m}^{r s}-\frac{7}{10} \tilde{\gamma}_{k(i} \tilde{\Gamma}^{k}{ }_{j\rangle}+\frac{7}{30} \tilde{\gamma}_{i j} \tilde{\Gamma}^{k}{ }_{, k}\right. \\
& \left.+8 Q_{(i, j)}-\frac{8}{3} \tilde{\gamma}_{i j} \tilde{\gamma}^{k l} Q_{k, l}\right) \alpha e^{-4 \phi}+\ldots \\
\dot{K}= & -6 \alpha \tilde{\gamma}^{k l} Q_{k, l}+\ldots \\
\dot{\tilde{\Gamma}}^{i}= & -\frac{4}{3} \alpha \tilde{\gamma}^{i j} K_{, j}+\ldots \\
\dot{Q}_{i}= & -\frac{\alpha}{6} K,_{i}+\ldots \\
\dot{V}^{i j}{ }_{k}= & -2 \alpha(59 \mathrm{c}) \\
& +\ldots
\end{aligned}
$$

The new evolution equations (59f) and (59g) are obtained simply by taking a time derivative of the definitions (55a) and (55b) and substituting the evolution equations (8b) and (8a) in the resulting right-hand sides. No linear combinations with the constraints are used.

In order to see that this initial value problem is strongly hyperbolic in the santadard sense [12], the eigenvalue problem of the principal symbol in an arbitrary direction must be solved. This is equivalent to assuming that all the fields have a plane wave dependence of the form $\exp i\left(\xi_{k} x^{k}-s t\right)$ with real $s$ and arbitrary $\xi_{i}$ so that $\xi_{i} \xi_{j} \gamma^{i j}=1$. The eigenvalue problem of the principal symbol has thus the form:

$$
\begin{aligned}
s \tilde{\gamma}_{i j}= & 0 \\
s \phi= & 0 \\
s \tilde{A}_{i j}= & -\alpha e^{-4 \phi}\left(\frac{1}{2} \tilde{\gamma}_{i r} \tilde{\gamma}_{j s} \tilde{\gamma}^{k m} V^{r s}{ }_{k} \xi_{m}-\frac{7}{10} \tilde{\gamma}_{k(i} \xi_{j)} \tilde{\Gamma}^{k}\right. \\
& \left.+\frac{7}{30} \tilde{\gamma}_{i j} \tilde{\Gamma}^{k} \xi_{k}+8 Q_{(i} \xi_{j)}-\frac{8}{3} \tilde{\gamma}_{i j} \tilde{\gamma}^{k l} Q_{k} \xi_{l}\right) \\
s K= & -6 \alpha \tilde{\gamma}^{k l} Q_{k} \xi_{l} \\
s \tilde{\Gamma}^{i}= & -\frac{4}{3} \alpha \tilde{\gamma}^{i j} K \xi_{j} \\
s Q_{i}= & -\frac{\alpha}{6} K \xi_{i} \\
s V^{i j}= & -2 \alpha\left(\tilde{A}^{i j} \xi_{k}-\frac{3}{5} \delta^{(i} \tilde{A}^{j) s} \xi_{s}+\frac{1}{5} \tilde{\gamma}^{i j} \tilde{\gamma}_{k l} \tilde{A}^{l s} \xi_{s}\right)
\end{aligned}
$$

The reader can verify that this problem has the following eigenvalues:

- $s=0$ with 18 eigenvectors labeled by free values of $\tilde{\gamma}_{i j}, \phi, \tilde{\Gamma}^{i}$, the two components of $Q_{i}$ except $\gamma^{k l} Q_{k} \xi_{l}$ and the seven components of $V^{i j}{ }_{k}$ other than $\gamma^{k l} V^{i j}{ }_{k} \xi_{l}$.

- $s= \pm \alpha$ with eigenvectors given by

$$
\begin{aligned}
\tilde{\gamma}_{i j} & =0 \\
\phi & =0 \\
\tilde{\Gamma}^{i} & =-\frac{4 \alpha}{3 s} e^{4 \phi} \xi^{i} K \\
Q_{i} & =-\frac{\alpha}{6 s} \xi_{i} K \\
\tilde{A}^{i j} \xi_{i} & =\frac{2 s}{3} e^{8 \phi} \xi^{i} K \\
V^{i j}{ }_{k} & =\frac{4 \alpha}{5}\left(\delta^{(i} \xi^{j)}-\frac{1}{3} \gamma^{i j} \xi_{k}\right) e^{4 \phi} K-\frac{2 \alpha}{s} \tilde{A}^{i j} \xi_{k}
\end{aligned}
$$

with free values of $K$ and of the two components of $\tilde{A}^{i j}$ other than $\tilde{A}^{i j} \xi_{j}$. These are six eigenvectors in all: three for $s=\alpha$ and three for $s=-\alpha$.

- $s= \pm \alpha \sqrt{3 / 5}$ with eigenvectors given by

$$
\begin{aligned}
\tilde{\gamma}_{i j} & =0 \\
\phi & =0 \\
\tilde{\Gamma}^{i} & =0 \\
Q_{i} & =0 \\
K & =0 \\
\tilde{A}^{i j} & =\frac{3}{2}\left(\xi^{i} \xi^{j}-\frac{1}{3} \gamma^{i j}\right) \tilde{A}^{k l} \xi_{k} \xi_{l} \\
V^{i j}{ }_{k} & =\frac{\alpha}{s}\left(\left(3 \xi^{i} \xi^{j}-\gamma^{i j}\right) \xi_{k}+\frac{6}{5} \delta_{k}^{(i} \xi^{j)}\right) \tilde{A}^{k l} \xi_{k} \xi_{l}
\end{aligned}
$$

with free values of the component $\tilde{A}^{k l} \xi_{k} \xi_{l}$. These are two eigenvectors in all: one for $s=\alpha \sqrt{3 / 5}$ and one for $s=-\alpha \sqrt{3 / 5}$. 
- $s= \pm \alpha \sqrt{7 / 10}$ with eigenvectors given by

$$
\begin{aligned}
\tilde{\gamma}_{i j}= & 0 \\
\phi= & 0 \\
\tilde{\Gamma}^{i}= & 0 \\
Q_{i}= & 0 \\
K= & 0 \\
\tilde{A}^{i j}= & 2 \xi^{(i} \tilde{A}^{j) m} \xi_{m} \\
V^{i j}= & -\frac{\alpha}{s}\left(4 \xi^{(i} \tilde{A}^{j) m} \xi_{m} \xi_{k}-\frac{12}{5} \delta_{k}^{(i} \tilde{A}^{j) m} \xi_{m}\right. \\
& \left.+\frac{2}{5} \tilde{\gamma}^{i j} \tilde{\gamma}_{k l} \tilde{A}^{l m} \xi_{m}\right)
\end{aligned}
$$

with free values of the two components $\tilde{A}^{i j} \xi_{j}$ other than $\tilde{A}^{k l} \xi_{k} \xi_{l}$ (which vanishes). These are four eigenvectors in all: two for $s=\alpha \sqrt{7 / 10}$ and two for $s=-\alpha \sqrt{7 / 10}$.

In all of the above, as in what follows, we are using the notation $\xi^{i} \equiv \gamma^{i j} \xi_{j}$.

All eigenvalues are thus real and there are $18+6+$ $2+4=30$ linearly independent eigenvectors. The initial value problem of Eqs. (59) is, thus, strongly hyperbolic in the standard sense, and has six pairs of "non-static" characteristic fields traveling with non-zero speeds. Not all characteristic speeds are equal to the speed of light, though: there are only three pairs of incoming-outgoing characteristic fields traveling at the speed of light. The other three pairs of "non-static" characteristic fields travel at subluminal speeds. (This does not necessarily contradict [15], since in that reference the authors use a different first-order reduction of the BSSN equations.) In the following we write down explicitly the characteristic fields with non-vanishing characteristic speeds, with the aim of relating some of them to the boundary equations of Section III

The three pairs of characteristic fields that travel at light speed $s= \pm \alpha$ are

$$
\begin{aligned}
{ }^{ \pm} U^{K} \equiv & K \pm 6 \xi^{k} Q_{k} \\
{ }^{ \pm} U^{i j} \equiv & \tilde{A}^{i j}-2 \xi^{(i} \tilde{A}^{j) k} \xi_{k}+\frac{1}{2}\left(\xi^{i} \xi^{j}+\gamma^{i j}\right) \tilde{A}^{k l} \xi_{k} \xi_{l} \\
& \pm\left(-\frac{1}{2} V^{i j}{ }_{k} \xi^{k}+\xi^{(i} V^{j) l}{ }_{k} \xi_{l} \xi^{k}\right. \\
& \left.-\frac{1}{4}\left(\xi^{i} \xi^{j}+\gamma^{i j}\right) V^{m l}{ }_{k} \xi_{m} \xi_{l} \xi^{k}\right)
\end{aligned}
$$

Notice that ${ }^{ \pm} U^{i j} \tilde{\gamma}_{i j}={ }^{ \pm} U^{i j} \xi_{j}=0$. As observed previously, they are labeled by free values of $K$ and of the two components of $\tilde{A}^{i j}$ other than the projections along the characteristic direction $\xi_{i}$.

The pair of characteristic fields with $s= \pm \alpha \sqrt{3 / 5}$ is

$$
\begin{aligned}
{ }^{ \pm} U \equiv & \tilde{A}^{i j} \xi_{i} \xi_{j}-\frac{2}{3} e^{4 \phi} K \pm \sqrt{\frac{5}{3}}\left(-\frac{1}{2} V_{k}^{m l} \xi_{m} \xi_{l} \xi^{k}\right. \\
& \left.+\frac{7}{15} \tilde{\Gamma}^{i} \xi_{i}-\frac{4}{3} e^{4 \phi} \xi^{k} Q_{k}\right)
\end{aligned}
$$

As observed before, it is labeled by the projection $\tilde{A}^{i j} \xi_{i} \xi_{k}$.

The two pairs of characteristic fields with $s=$ $\pm \alpha \sqrt{7 / 10}$ are

$$
\begin{aligned}
{ }^{ \pm} U^{i} \equiv & \tilde{A}^{i j} \xi_{j}-\xi^{i} \tilde{A}^{k l} \xi_{k} \xi_{l} \pm \frac{1}{2} \sqrt{\frac{7}{10}}\left(\tilde{\Gamma}^{i}-\xi^{i} \tilde{\Gamma}^{k} \xi_{k}\right. \\
& \left.-V^{i j}{ }_{k} \xi_{j} \xi^{k}+\xi^{i} V^{m l}{ }_{k} \xi_{m} \xi_{l} \xi^{k}\right) \\
& \pm 4 \sqrt{\frac{10}{7}}\left(-\tilde{\gamma}^{i k} Q_{k}+e^{4 \phi} \xi^{i} \xi^{k} Q_{k}\right)
\end{aligned}
$$

Notice that $U^{i} \xi_{i}=0$. These are labeled by the two projections $\tilde{A}^{i j} \xi_{j}$ other than $\tilde{A}^{k l} \xi_{k} \xi_{l}$, as anticipated previously.

In order to see which of these fields are prescribed by the boundary equations (18), (19), (24) and (26), we restrict attention to $\xi_{i}=(1,0,0) / \sqrt{\gamma^{x x}}$. Lowering the in$\operatorname{dex} i$ with $\tilde{\gamma}_{i j}$ we have

$$
\begin{aligned}
& { }^{ \pm} U_{y}=\frac{\tilde{A}_{y}^{x}}{\sqrt{\gamma^{x x}}} \pm \frac{1}{2} \sqrt{\frac{7}{10}} \tilde{\gamma}_{y k}\left(\tilde{\Gamma}^{k}-V^{x k} l \frac{\gamma^{x k}}{\gamma^{x x}}\right) \mp 4 \sqrt{\frac{10}{7}} Q_{y} \\
& { }^{ \pm} U_{z}=\frac{\tilde{A}_{z}^{x}}{\sqrt{\gamma^{x x}}} \pm \frac{1}{2} \sqrt{\frac{7}{10}} \tilde{\gamma}_{z k}\left(\tilde{\Gamma}^{k}-V^{x k}{ }_{l} \frac{\gamma^{x k}}{\gamma^{x x}}\right) \mp 4 \sqrt{\frac{10}{7}} Q_{z}
\end{aligned}
$$

Inverting for $\tilde{A}_{y}^{x}$ and $\tilde{A}_{z}^{x}$ we have

$$
\begin{aligned}
& \tilde{A}_{y}^{x}=\frac{\sqrt{\gamma^{x x}}}{2}\left({ }^{-} U_{y}+{ }^{+} U_{y}\right) \\
& \tilde{A}_{z}^{x}=\frac{\sqrt{\gamma^{x x}}}{2}\left({ }^{-} U_{z}+{ }^{+} U_{z}\right)
\end{aligned}
$$

Clearly, the boundary equations (18) and (19) prescribe the time derivatives of the incoming fields ${ }^{-} U_{y}$ and ${ }^{-} U_{z}$, respectively, in terms of their outgoing counterparts.

Next, with $\xi_{i}=(1,0,0) / \sqrt{\gamma^{x x}}$, the fields ${ }^{ \pm} U$ read

$$
\begin{aligned}
{ }^{ \pm} U \equiv & \frac{\tilde{A}^{x x}}{\gamma^{x x}}-\frac{2}{3} e^{4 \phi} K \pm \sqrt{\frac{5}{3}}\left(-\frac{1}{2} V_{k}^{x x} \frac{\gamma^{k x}}{\left(\gamma^{x x}\right)^{\frac{3}{2}}}\right. \\
& \left.+\frac{7}{15} \frac{\tilde{\Gamma}^{x}}{\sqrt{\gamma^{x x}}}-\frac{4}{3} e^{4 \phi} Q_{k} \frac{\gamma^{k x}}{\sqrt{\gamma^{x x}}}\right)
\end{aligned}
$$

which can be inverted as

$$
\tilde{A}^{x x}-\frac{2}{3} \tilde{\gamma}^{x x} K=\frac{\gamma^{x x}}{2}\left({ }^{-} U+{ }^{+} U\right)
$$

Furthermore, since $\tilde{A}^{x x}-\frac{2}{3} \tilde{\gamma}^{x x} K=\tilde{\gamma}^{x j}\left(\tilde{A}_{j}^{x}-\frac{2}{3} \delta_{j}^{x} K\right)$ then we have:

$$
\tilde{\gamma}^{x j}\left(\tilde{A}_{j}^{x}-\frac{2}{3} \delta_{j}^{x} K\right)=\frac{\gamma^{x x}}{2}\left({ }^{-} U+{ }^{+} U\right)
$$

Isolating the term with $j=x$ we have

$$
\tilde{\gamma}^{x x}\left(\tilde{A}_{x}^{x}-\frac{2}{3} K\right)=\frac{\gamma^{x x}}{2}\left({ }^{-} U+{ }^{+} U\right)-\tilde{\gamma}^{x y} \tilde{A}_{y}^{x}-\tilde{\gamma}^{x z} \tilde{A}_{z}^{x}
$$


Using (66) in the right-hand side, we finally have

$$
\begin{aligned}
\tilde{A}_{x}^{x}-\frac{2}{3} K= & \frac{e^{4 \phi}}{2}\left({ }^{-} U+{ }^{+} U\right)-\frac{\tilde{\gamma}^{x y}}{2 \tilde{\gamma}^{x x}}\left({ }^{-} U_{y}+{ }^{+} U_{y}\right) \\
& -\frac{\tilde{\gamma}^{x z}}{2 \tilde{\gamma}^{x x}}\left({ }^{-} U_{z}+{ }^{+} U_{z}\right)
\end{aligned}
$$

Clearly, since the time derivatives of the incoming fields ${ }^{-} U_{y}$ and $-U_{z}$ are already prescribed by (18) and (19), then the boundary equation (24) prescribes the time derivative of the incoming field ${ }^{-} U$ in terms of its outgoing counterpart.

It only remains to understand the role of the fourth boundary equation (26). The reader can verify that the combination

$$
{ }^{0} U \equiv \tilde{\Gamma}^{x}-8 \tilde{\gamma}^{x k} Q_{k}
$$

is one of the 18 characteristic fields with zero characteristic speed. But this is exactly equal to the combination that appears under the time-derivative sign in (26) up to terms of lower order, that is

$$
\tilde{\Gamma}^{x}-8 \tilde{\gamma}^{x k} Q_{k}=\gamma^{x i}\left(\left(\ln \left(\operatorname{det} \gamma_{k l}\right)\right),{ }_{i}-\gamma^{k l} \gamma_{i k, l}\right)+\ldots
$$

Clearly, thus, the fourth boundary equation (26) is a condition on the time derivative of the "static" characteristic field ${ }^{0} U$, and, as such, it should be identically satisfied. The fourth equation is not necessary in order to prescribe incoming fields.

In summary, we have found that three of the six incoming characteristic fields are prescribed by three of the four equations $G_{a}^{x}=0$. These are precisely the characteristic fields that travel at speeds different from the speed of light. The fourth equation is redundant, but must be satisfied by a static characteristic field as a consequence of the initial data and the evolution equations.

This is entirely consistent with the analysis of Section IV The results of this Section show that, in the case of the first-order reduction, there are only three boundary prescriptions in the set $G_{a}^{x}=0$, and the three incoming fields that travel at the speed of light remain freely specifiable. This is very similar to the case of the EinsteinChristoffel formulation discussed in [2] and [3]. The fields that remain freely specifiable are labelled by the components $\tilde{A}_{z}^{y}$ and $\tilde{A}_{y}^{y}-\tilde{A}_{z}^{z}$, and the trace $K$ of the extrinsic curvature, as in the case of the Einstein-Christoffel formulation. The exception is that in the present case it becomes clear that the incoming fields that remain free are physical in the sense that they are the only ones traveling at the speed of light (the same case cannot be made in the Einstein-Christoffel formulation because all its incoming fields travel at light speed).

It is not clear to us whether any of the conclusions regarding the incoming characteristic fields may be directly transferred from the first-order reduction to the original BSSN equations, that is, Eqs. (8). The main use of the results of this Section is to add insight into the boundary-value problem of the (standard) BSSN equations, as well as to increase our understanding of the role of the Einstein boundary conditions $G_{a}^{x}=0$ in the initialboundary-value problem of the Einstein equations across formulations.

\section{CONCLUDING REMARKS}

We have written down explicitly the principal terms of the four components of the projection of the Einstein tensor perpendicularly to a timelike boundary in terms of the variables of the BSSN formulation, Eqs. (8). The resulting expressions, Eqs. (18), (19), (24) and (26), contain no second derivatives across the boundary of any of the fundamental variables, and no first derivatives across the boundary of any of the three first-order variables $\tilde{\Gamma}^{i}$. Consequently, setting them to zero results in four equations internal to the boundary in the sense that they are constraints on the timelike boundary surface. Such boundary equations can then be interpreted as boundary conditions for the BSSN formulation of the Einstein equations. Trivially, these boundary conditions are necessary to the effect of satisfying the Einstein equations on the boundary surface. But far from trivially, we have demonstrated that three of them are necessary in order to ensure that the solution of the BSSN equations will satisfy the constraints in the future of the initial slice.

A meaning to the three non-trivial boundary equations can be assigned as follows. A timelike boundary surface can be interpreted as an artificial boundary separating two regions in a spacetime generated by the same Cauchy surface (that is: a "big" spacetime with no boundary or with a null boundary). In this interpretation, the values on the timelike "boundary" surface are completely determined by evolution from the initial data. The initial data satisfy the momentum and Hamiltonian constraints. Therefore, the values on the timelike "boundary" surface must be related by some sort of evolution of the constraints from their initial values. Three of the constraints propagate towards the "boundary" from data in the interior region of the Cauchy surface. But three of them propagate towards the "boundary" from Cauchy data in the other side of the timelike surface. These three "incoming" constraints must affect the "boundary" values that are interpreted as incoming, and a prescription for their effect on the boundary values is needed. The point is that the three nontrivial equations in the set $G_{a}^{x}=0$ accomplish such a prescription by realizing, in a sense, the evolution of the incoming initial constraints towards the "boundary" surface.

Just like in the case of the ADM equations, the problem of the boundary conditions of the BSSN formulation remains open in the following sense. There are, in principle, 15 variables whose boundary values are needed in order to proceed forward in an integration in time. Of these, four can be obtained from initial data by the four equations $G_{a}^{x}=0$, which are, in fact, time-evolution equations internal to the boundary surface. It is not known, at this time, how many of the remaining 11 variables are freely 
specifiable at the boundary, and how many are determined by evolution from initial data. The potentially freely specifiable variables represent an indeterminacy in the sense that their role in shaping up the final solution is as important as that of the initial data. Thus, physical insight would be required to prescribe the (potentially free) boundary data in a manner analogous to the physical insight required to determine the free initial data for a particular problem (e.g, for binary black hole collisions).

We have also studied the related problem of a stronglyhyperbolic reduction of the BSSN equations, that is, Eqs. (59). In this case, the boundary values that need to be specified are known to be those of six of the 30 fundamental variables, of which three are prescribed by the Einstein boundary conditions. The others are determined, in principle, from the initial values and the evolution equations. We have not concerned ourselves with the question of determining which linear combinations of $G_{a}^{x}=0$ lead to a well-posed initial-boundary-value problem, since our present first-order reduction is meant only as an auxiliary device in order to shed light on the original problem and is not expected to be used for numerical simulations. The problem of separating the Einstein boundary conditions for this first-order reduction into a well-posed set and an ill-posed set remains open at this time.
Other issues that remain wide open are whether or not the (standard) BSSN formulation with the Einstein boundary conditions can be implemented in a numerically stable manner in the rigorous sense of finitedifferences, and whether the Einstein boundary conditions have any effect on the run time of numerical simulations. Because the BSSN formulation is not strongly hyperbolic in the standard sense, the former issue lies outside of our current interests. The latter issue is also of fundamental importance to numerical simulations because it has been observed that constraint violations play a crucial role in shortening the run time [16]. Our expectation is that the Einstein boundary conditions, being "constraint-preserving" in the sense discussed in Section IV can potentially increase the run time of numerical simulations, assuming that other potential numerical instabilities are under control.

\section{Acknowledgments}

This work was supported by the NSF under grants No. PHY-0244752 to Duquesne University, and No. PHY0135390 to Carnegie Mellon University.
[1] S. Frittelli and R. Gómez, Class. Quantum Grav. 20, 2379 (2003), gr-qc/0302032.

[2] S. Frittelli and R. Gómez, Phys. Rev. D 68, 044014 (2003), gr-qc/0302071.

[3] S. Frittelli and R. Gómez, Einstein boundary conditions in relation to constraint propagation for the initial-boundary value problem of the Einstein equations, gr-qc/0310064.

[4] A. Anderson and J. W. York, Phys. Rev. Lett. 82, 4384 (1999).

[5] J. W. York, in Sources of Gravitational Radiation, edited by L. Smarr (Cambridge University Press, Cambridge, 1979).

[6] T. W. Baumgarte and S. L. Shapiro, Phys. Rev. D 59, 024007 (1999).

[7] M. Shibata and T. Nakamura, Phys. Rev. D 52, 5428 (1995).

[8] S. Frittelli and O. A. Reula, Phys. Rev. Lett. 76, 4667 (1996).

[9] U. Sperhake, K. L. Smith, B. Kelly, P. Laguna, and
D. Shoemaker, Impact of densitized lapse slicings on evolutions of a wobbling black hole, gr-qc/0307015.

[10] B. Bruegmann, W. Tichy, and N. Jansen, Numerical simulation of orbiting black holes, gr-qc/0312112.

[11] P. Laguna and D. Showmaker, Class. Quantum Grav. 19, 3679 (2002).

[12] B. Gustaffson, H.-O. Kreiss, and J. Oliger, Timedependent problems and difference methods (Wiley, New York, 1995).

[13] G. Nagy, O. Ortiz, and O. A. Reula, Strongly hyperbolic second order Einstein's evolution equations, gr-qc/0402123.

[14] S. Frittelli and O. A. Reula, J. Math. Phys. 40, 5143 (1999).

[15] O. Sarbach, G. Calabrese, J. Pullin, and M. Tiglio, Phys. Rev. D 66, 064002 (2002).

[16] L. E. Kidder, M. A. Scheel, S. A. Teukolsky, E. D. Carlson, and G. B. Cook, Phys. Rev. D 62, 084032 (2000). 